\title{
Sífilis em gestantes no Acre: uma análise do período compreendido entre 2015 a 2020
}

\author{
Syphilis in pregnant women in Acre: an \\ analysis of the period from 2015 to 2020
}

\author{
Gerlane Silva Cavalcante ${ }^{1}$ (1) \\ Maria Dulcilene do Nascimento Araujo de Paula² (1) \\ Natiele Silva Nascimento ${ }^{3}$ (1) \\ Matilde da Silva Conceição ${ }^{4}$ (1) \\ Christopher Wando da Silva Souza ${ }^{5}$ \\ Ruth Silva Lima da Costa ${ }^{6}$ (1)
}

1-5 União Educacional do Norte (Rio Branco). Acre, Brasil. gerlanegeovane12@gmail.com, dulcymaria2011@hotmail.com, natiellena@gmail.com,
matieconceicao@gmail.com, christopherwando07@gmail.com
${ }^{6}$ Autora para correspondência. União Educacional do Norte (Rio Branco). Acre, Brasil.ruttylyma@gmail.com

RESUMO | OBJETIVO: Descrever os casos de sífilis em gestantes no estado do Acre no período compreendido entre 2015 a 2020. MÉTODO: Estudo ecológico descritivo com a utilização de dados secundários disponíveis no site do Departamento de Informações do Sistema Único de Saúde - DATASUS, tabulados a partir do TABNET. As variáveis utilizadas foram: ano de notificação, grau de instrução, faixa etária, raça/cor, classificação clínica, idade gestacional e esquema de tratamento. RESULTADOS: Foram notificados 2443 casos no período. A maioria encontrava-se na faixa etária entre 20 a 29 anos, 1146 (47\%), com ensino fundamental incompleto, 677 (28\%), e raça/cor parda, 1983 (81\%). A doença apresentou seu pico mais elevado no ano de 2018 , com a notificação de 627 (26\%) casos, e uma redução partir deste ano. A maior parte foi classificada como sífilis primária 900 (37\%), diagnosticadas no $1^{\circ}$ trimestre da gravidez 876 (36\%). Quanto ao esquema de tratamento, 1891 (77,4\%) estavam sem informação no sistema, e a penicilina foi o antibiótico de escolha de 529 (21,7\%). CONCLUSÃo: Os casos de sífilis no Acre apresentaram redução no último ano analisado, o que pode estar relacionado a baixa frequência ao pré-natal, em decorrência da pandemia de Covid-19. Foram acometidas mulheres jovens e classificadas com sífilis primária no $1^{\circ}$ trimestre da gravidez.

PALAVRAS-CHAVE: Sífilis. Infecções por treponemal. Gestantes. Notificação de Doenças.
ABSTRACT | OBJECTIVE: To describe cases of syphilis in pregnant women in the state of Acre from 2015 to 2020. METHOD: This is a descriptive ecological study using secondary data available on the website of the Information Department of the Unified Health System - DATASUS, tabulated from the TABNET whose variables used were: year of notification, level of education, age group, race/color, clinical classification, gestational age, and treatment regimen. RESULTS: 2443 cases were reported in the period, and most were aged between 20 and 29 years, 1146 (47\%), with incomplete primary education 677 (28\%) and brown race/color 1983 (81\%). The disease had its highest peak in 2018, with the notification of 627 (26\%) cases, showing a reduction from this year. As for the clinical classification, most were classified as primary syphilis 900 (37\%), diagnosed in the 1 st trimester of pregnancy 876 (36\%). As for the treatment scheme used, the one without information in the system predominated 1891 (77.4\%), and among the available data, penicillin was the antibiotic of choice 529 (21.7\%). CONCLUSION: it was found that the cases of syphilis in Acre showed a reduction in the last year analyzed, which may be related to the low frequency of prenatal care due to the Covid-19 pandemic. Young women were affected and classified as primary syphilis in the 1st trimester of pregnancy.

KEYWORDS: Syphilis. Treponemal Infections. Pregnant. Notification of Diseases. 


\section{Introdução}

A sífilis é uma doença infecciosa, de caráter sistêmico e evolução crônica, sujeita a períodos de latência e surtos de agudização. 1 É uma doença bacteriana causada pelo Treponema Pallidum, uma bactéria gram-negativa do grupo das espiroquetas, podendo ocorrer transmissão sexual ou vertical e causar respectivamente a forma adquirida ou congênita da doença.?

A transmissão vertical da sífilis, denominada sífilis congênita é um dos casos de maior relevância na saúde pública atualmente e sua preeminência de transmissão a nível global faz com que as taxas de contaminação não declinem, apesar da implementação de medidas preventivas e educativas. 3

Segundo dados a Organização Mundial de Saúde (OMS), aproximadamente 1,5 milhão de mulheres grávidas são infectadas com sífilis todos os anos, e a maioria delas não é tratada, levando à contaminação dos filhos, com resultados adversos, tais como óbito neonatal, baixo peso ao nascer e/ou evidência clínica de infecção. ${ }^{4}$

No Brasil, somente em 2019, foram notificados no Sistema Nacional de Agravos de Notificação (SINAN) 152.915 casos de sífilis adquirida; 61.127 casos de sífilis em gestantes; 24.130 casos de sífilis congênita; e 173 óbitos por sífilis congênita (taxa de mortalidade de $5,9 / 100.000$ nascidos vivos). ${ }^{5}$

A sífilis em gestantes é doença de notificação compulsória no Brasil desde 2005, através da portaria $\mathrm{n}^{\circ}$. $33 / 2005$, e a sífilis congênita desde 1986, pela portaria $n^{\circ} .542 / 1986$, tornando-se então obrigatória a comunicação à autoridade de saúde quando houver confirmação da doença..

Ressalta-se que dentre os fatores considerados determinantes a riscos associados à transmissão vertical da doença estão: pré-natal iniciado tardiamente, número reduzido de consultas, não realização de testes para sífilis ou não realização do tratamento dos parceiros sexuais e da gestante. ${ }^{?}$

Nesse sentido, torna-se prioridade a prevenção da transmissão vertical, através da realização do prénatal, com a testagem e tratamento em tempo hábil, além do tratamento dos parceiros, bem como o tratamento profilático com dose única penicilina em todos os recém-nascidos de mães com diagnóstico de sífilis na gestação. $\underline{\underline{6} .8}$

Destarte, é muito importante que ocorra a vigilância da sífilis na gestação e da sífilis congênita durante a assistência à mulher, que tem como objetivo identificar os casos para subsidiar ações de prevenção e controle, monitorar o perfil epidemiológico e suas tendências, acompanhar e avaliar as ações para à sua eliminação. ${ }^{2}$

No Acre, há uma escassez de estudos frente a essa temática, evidenciando assim a necessidade da realização do presente estudo cujo objetivo é descrever os casos de sífilis em gestantes no Acre no período compreendido entre 2015 a 2020.

\section{Método}

Trata-se de um estudo ecológico descritivo com a utilização de dados secundários extraídos no site do Departamento de Informática do SUS - DATASUS, tabulados a partir do TABNET utilizando os dados de "Epidemiológicas e Morbidade" do Sistema Nacional de Agravos de Notificação (SINAN NET), através dos seguintes passos: DATASUS; Acesso à Informação; Informações em Saúde (TABNET); epidemiológicas e Morbidade $\rightarrow$ Sífilis em gestantes.

Para a coleta de dados, foram analisadas variáveis como: ano de notificação, grau de instrução, faixa etária, raça/cor, classificação clínica, idade gestacional e esquema de tratamento. A amostra foi composta por todos os casos notificados sífilis em gestantes e inseridos na plataforma DATASUS no período de 2015 a 2020, totalizando 2443 casos.

Foram incluídos no estudo os dados de casos notificados sífilis em gestantes disponíveis na plataforma do DATASUS, no período de 2015 a 2020, de acordo com as variáveis do estudo: ano de notificação, grau de instrução, faixa etária, raça/cor, classificação clíni$\mathrm{ca}$, idade gestacional e esquema de tratamento. Não foram excluídos nenhum dos dados. A coleta de dados foi realizada em março de 2021. 
Os dados quantificados foram apresentados em frequência absoluta e percentual. Foram demonstrados em forma de tabelas e gráficos de acordo com as variáveis existentes. Para produção dos gráficos foi utilizada a ferramenta do Microsoft Office Excel 2010.

O trabalho não foi submetido ao Comitê de Ética em Pesquisa - CEP local, por tratar-se de estudo em fontes secundárias e não se enquadrar dentro da legislação do CONEP/MS, resolução de 466/2012.

\section{Resultados}

No período de estudo, foram notificados 2443 casos de sífilis em gestantes no estado do Acre. A categorização das amostras sob os parâmetros de ano de notificação, grau de instrução, faixa etária, raça/cor, estão evidenciados na tabela 1. Referente aos dados sócios demográficos das gestantes acometidas com sífilis no estado no Acre no período de 2015 a 2020, a maioria encontrava-se na faixa etária compreendida entre 20 a 29 anos, 1146 (47\%). Quanto ao grau de escolaridade, a maior parte possuía o ensino fundamental incompleto, 677 (28\%), seguido do ensino médio completo, 611(25\%), e frente à questão de raça/cor, predominou a parda, 1983 (81\%).

Tabela 1. Perfil sóciodemográfico das gestantes com sífilis no estado do Acre, no período de 2015 a 2020 (n= 2443$)$

\begin{tabular}{lcc}
\hline \multicolumn{1}{c}{ VARIÁVEL } & $\mathbf{N}$ & $\mathbf{\%}$ \\
\hline Faixa Etária & 47 & 2,0 \\
10 a 14 anos & 811 & 33,0 \\
15 a 19 anos & 1146 & 47,0 \\
20 a 29 anos & 396 & 16,0 \\
30 a 39 anos & 43 & 2,0 \\
40 anos ou mais & & 2,0 \\
Grau de Escolaridade & 37 & 28,0 \\
Analfabeta & 677 & 9,0 \\
Fundamental Incompleto & 209 & 20,0 \\
Fundamental Completo & 498 & 24,0 \\
Médio Incompleto & 611 & 4,0 \\
Médio Completo & 98 & 2,0 \\
Superior Incompleto & 51 & 11,0 \\
Superior Completo & 37 & 9,0 \\
Ignorado & & 5,0 \\
Cor/raça & 222 & 1,0 \\
Branca & 110 & 81,0 \\
Preta & 29 & 2,0 \\
Amarela & 1983 & 2,0 \\
Parda & 56 & \\
Indígena & 43 & \\
Ignorada & &
\end{tabular}

Fonte: DATASUS, 2021

Com relação ao número de casos diagnosticados por ano, a doença no estado apresentou em 2018 a notificação de 627, uma redução dos casos registrados após este período. 
Figura 1. Número de casos de sífilis em gestantes no estado do Acre, no período de 2015 a 2020 (n= 2443)

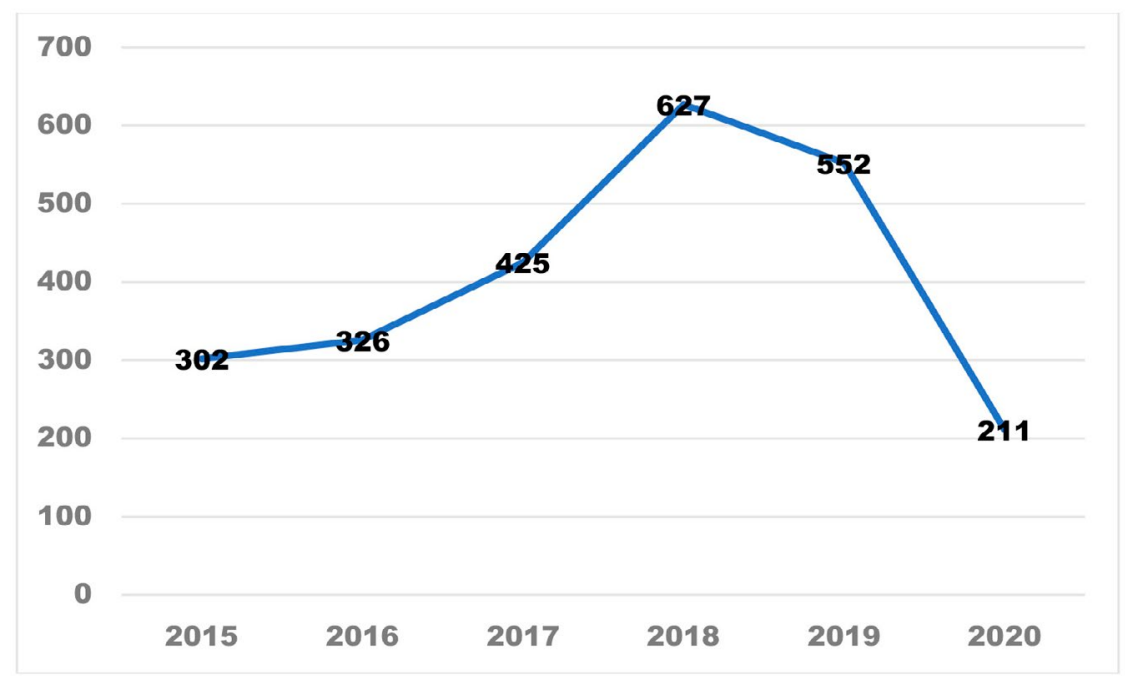

Fonte: DATASUS, 2021

Na tabela 2, estão descritas as características clínicas da doença em gestantes no Acre. Observa-se que a maioria foi classificada como sífilis primária com 900 (37\%) dos casos, seguido pela sífilis latente, com 858 (35\%) dos casos. No que tange à idade gestacional no momento do diagnóstico, a maior parte ocorreu no $1^{\circ}$ trimestre da gravidez ,876 (36\%). Quanto ao esquema de tratamento utilizado, predominou sem informação, 1891 (77,4\%), sendo que dentre os dados disponíveis, a penicilina foi o antibiótico de escolha com 529(21,7\%) dos casos.

Tabela 2. Características clínicas da sífilis em gestantes no estado do Acre, no período de 2015 a 2020 (n=2443)

\begin{tabular}{lcc}
\hline \multicolumn{1}{c}{ VARIÁVEL } & $\mathbf{N}$ & $\mathbf{\%}$ \\
\hline Classificação Clínica & & 36,0 \\
Sífilis Primária & 900 & 6,0 \\
Sífilis Secundária & 137 & 12,0 \\
Sífilis Terciária & 283 & 35,0 \\
Sífilis Latente & 858 & 11,0 \\
Ignorado & 265 & 36,0 \\
Idade Gestacional & & 32,0 \\
$1^{\circ}$ Trimestre & 876 & 31,0 \\
$2^{\circ}$ Trimestre & 774 & 1,0 \\
$3^{\circ}$ Trimestre & 765 & \\
Idade gestacional ignorada & 28 & 21,6 \\
Esquema de Tratamento & & 0,2 \\
Penicilina & 529 & 0,5 \\
Outro Esquema & 4 & 0,4 \\
Não realizado & 12 & 77,4 \\
Ignorado & 7 & \\
Sem Informação & 1891 & \\
\hline Fon & & \\
\hline
\end{tabular}

Fonte: DATASUS, 2021

\section{Discussão}

Com relação às faixas etárias encontradas nesse estudo, corroboram os achados de Silva et al. $\stackrel{10}{ }$, nos quais há resultado muito semelhante, visto que grande parte $(49,67 \%)$ das gestantes diagnosticadas com sífilis tinham entre 21 e 30 anos. Outros estudos com resultados similares são o de Marques et al. $\stackrel{11}{ }$, no qual $74,9 \%$ tinham entre 20 e 39 anos. 
No que concerne à escolaridade, o estudo de Cavalcante, Pereira e Castro ${ }^{12}$, realizado em Tocantins, observou que $76 \%$ das gestantes com sífilis tinham escolaridade entre o ensino fundamental incompleto e o ensino médio completo, resultado semelhante com os $82 \%$ encontrados no presente estudo. Marques et al. ${ }^{11}$ justificam tal achado pelo fato de que mulheres de baixa escolaridade têm difícil acesso a informações sobre a doença e maior dificuldade para realização do pré-natal.

No que concerne à escolaridade, o estudo de Cavalcante, Pereira e Castro ${ }^{12}$, realizado em Tocantins, observou que $76 \%$ das gestantes com sífilis tinham escolaridade entre o ensino fundamental incompleto e o ensino médio completo, resultado semelhante com os $82 \%$ encontrados no presente estudo. Tal achado pode ser justificado devido mulheres de baixa escolaridade ter mais dificuldade ao acesso às informações sobre a doença e para realização do pré-natal.

Outrossim, no que se refere à raça/cor autodeclarada, no estudo de Marques et al.11, $80,3 \%$ das gestantes diagnosticadas com sífilis se autodeclararam pardas, resultado semelhante ao do presente estudo. Mulheres pardas e negras enfrentam dificuldades em relação à assistência à saúde, tendo em vista que elas são vítimas de desigualdade no acesso ao pré-natal adequado, de modo que contam com menor número de consultas e exames, além de se vincularem menos à maternidade para o parto e receberem menos orientações. $\underline{\underline{13}}$

No que se refere ao número de casos notificados por ano, observou-se que partir do ano de 2015, começou a ocorrer o aumento do número de notificação de casos de sífilis, ao longo da série histórica estudada. Este fato pode estar atribuído a diversos fatores, como diminuição do número de sub-registros e, consequentemente, o aumento das notificações, bem como ações de vigilância epidemiológica direcionadas para uma melhor abordagem e identificação dos casos da doença no município. ${ }^{14}$

No entanto, chama-se atenção para uma expressiva redução no ano de 2020, que pode estar relacionada com o fato do país encontrar-se em pandemia da Covid-19, e justificar a ausência do comparecimento as consultas de pré-natal e a consequente falta de notificação da doença. Corroborando os achados da presente pesquisa, um estudo exploratório sobre as taxas de incidência de sífilis e gonorreia durante a pandemia de Covid-19, realizado em Cuba, evidenciou que após a confirmação da doença e a implementação das medidas de confinamento social, as taxas de incidência de sífilis e gonorreia diminuíram significativamente. $\frac{15}{15}$

Destarte, um estudo realizado em uma unidade de tratamento de infecções sexualmente transmissíveis (ISTs) o instituto dermatológico San Gallicano, de Roma, também evidenciou que desde o início do lockdown, observou-se uma redução dos diagnósticos de IST, principalmente de sífilis, sendo assim, o medo da infecção pelo SARS-CoV-2, pode ter reduzido os encontros sexuais e levado a um declínio das infecções por sífilis. No entanto, não se pode excluir a possibilidade da contaminação, devido à não procura das unidades de saúde para realização do diagnóstico e tratamento. $\frac{16}{}$

Sendo assim, a limitação de consultas ambulatoriais e serviços de triagem hospitalar devido às medidas de isolamento social decorrentes da pandemia podem ter favorecido a circulação da sífilis e atrasado o diagnóstico e o tratamento adequado. Uma falsa sensação de proteção e contenção social gerada por medidas de bloqueio pode levar a subestimar a circulação de doenças sexualmente transmissíveis na comunidade. Principalmente o diagnóstico da sífilis requer um olhar perito e um alto nível de suspeição, como é característico da prática dermatológica de campo. ${ }^{17}$

Em conformidade com achados evidenciados na tabela 2 , no que se refere à classificação clínica da sífilis, estudo realizado na Bahia demonstrou que a sífilis primária também foi a mais notificada na região; entretanto, os registros evidenciaram que, em média, em pouco mais de $32 \%$ dos casos nesse estado não foi relatada a fase clínica da doença. $\underline{18}$

Vale ressaltar ainda que o fato de a sífilis gestacional do tipo primária ser a mais registrada na maioria das regiões brasileiras favorece uma boa resposta ao tratamento se instituído adequadamente e de maneira precoce. Todavia, a limitação de acesso a terapêutica preconizada, a penicilina $\mathrm{G}$. benzatina, sendo a única com alta eficácia para sífilis gestacional, assim como a não adesão ou descontinuidade por parte da paciente, impede que esses casos cursem de fato com um bom prognóstico, e predispõe um risco maior de transmissão vertical e diversos desfechos desfavoráveis.17,19 
No Brasil, é recomendado a solicitação de testes sorológico para detecção da doença em gestantes no pré-natal, no $1^{\circ}$ e $3^{\circ}$ trimestre para prevenção de agravos e instituição do tratamento adequado assim que possível, com o intuito de impedir a transmissão para o feto, além disso, deve-se realizar novos testes anteriormente ao parto. 20

No entanto em investigação realizada, $27 \%$ das gestantes que não realizaram o tratamento alegaram que o teste não treponêmico VDRL só positivou no segundo exame, às vésperas da entrada a maternidade. ${ }^{21}$ Esse tipo de situação soma-se ao fato de grávidas não fazerem o acompanhamento do pré-natal e as deficiências ainda presentes na assistência materno-fetais.22

No que diz respeito ao período da gestação em que foi diagnosticada a sífilis gestacional, Conceição et al. $\frac{23}{}$ identificaram que $46,3 \%$ do diagnóstico se deu no $3^{\circ}$ trimestre da gravidez no município de Caxias, enquanto no estudo de Souza et al. $\frac{18}{1}, 33 \%$ das notificações ocorreram no $2^{\circ}$ trimestre e $32 \%$ no terceiro trimestre; isso pode contribuir de maneira negativa para o aumento de casos da sífilis congênita, haja vista o tempo decorrido para a detecção da doença e início tardio do tratamento.

Ademais, para a terapêutica farmacológica da sífilis gestacional é disponibilizado a benzilpenicilina, variando a dose e a duração indicada de acordo com a classificação clínica da doença, este fármaco apresenta baixo custo e alta eficácia se aplicada corretamente, porém, houve um desabastecimento da matéria prima desse medicamento no Brasil, fator esse que pode estar diretamente relacionado à falha do tratamento assim como a manutenção e progressão da doença.

Nesse sentido, vale destacar que há persistência de indicação terapêutica não condizente com aquilo que está instituído como melhor fator de eficácia e possivelmente cura da doença, posto que Miranda et al..20 demostrou em uma pesquisa que cerca de $38 \%$ das pacientes no Distrito Federal e 54\% no Rio de Janeiro receberam outra terapia medicamentosa que não seja a penicilina benzatina e obtiveram, sucesso no tratamento.
Este estudo apresentou algumas limitações que merecem ser mencionadas, no que se refere ao o uso de dados secundários, que não permitem ao pesquisador controlar possíveis erros decorrentes de digitação e de registro, além de possíveis subnotificações. Apesar disto, acredita-se que, por se tratar de dados nacionais oficiais e de preenchimento obrigatório no serviço de saúde, os dados coletados permitiram o alcance dos objetivos propostos no presente estudo

\section{Conclusão}

Os achados do presente estudo evidenciaram que a sífilis gestacional se configura como um grave problema de saúde pública no estado, pois houve um expressivo número de notificações da doença no período de estudo, sendo evidenciada uma tendência de queda no último ano, mas que pode estar relacionada à pandemia de Covid 19 que assola o país e que pode estar levando à baixa adesão ao pré-natal e consequentemente menor número de diagnósticos e notificações da doença.

A maioria dos casos ocorreu em gestantes jovens, com o ensino fundamental incompleto e da raça/cor parda. Quanto à classificação clínica, a maioria foi classificada como sífilis primária e em gestantes no $1^{\circ}$ trimestre da gravidez, sendo a penicilina o antibiótico de escolha para o tratamento.

Nesse sentido, tendo em vista a relevância dos achados, reforça-se a necessidade de melhorias na assistência pré-natal para uma melhor adesão das gestantes e melhor atuação frente ao problema.

\section{Contribuições dos autores}

Cavalcante GS, Paula MDNA, Nascimento NS, Conceição MS e Souza CWS participaram da concepção, delineamento, coleta de dados da pesquisa, interpretação, busca e análise dados e redação do artigo científico. Costa RSL participou da concepção, delineamento, submissão do projeto ao Comitê de ética e pesquisa, interpretação dos dados, busca e análise dos dados da pesquisa, interpretação dos resultados e redação do artigo científico. 


\section{Conflito de interesses}

Nenhum conflito financeiro, legal ou político envolvendo terceiros (governo, empresas e fundações privadas, etc.) foi declarado para nenhum aspecto do trabalho submetido (incluindo, mas não se limitando a subvenções e financiamentos, participação em conselho consultivo, desenho de estudo, preparação de manuscrito, análise estatística, etc.).

\section{Referências}

1. Ministério da Saúde (Brasil). Guia de Vigilância em Saúde [Internet]. Brasília: Ministério da Saúde; 2016. Disponível em: https://portalarquivos2.saude.gov.br/images/pdf/2016/agosto/25/ GVS-online.pdf

2. Veronesi R, Focaccia R. Tratado de Infectologia. 5a. ed. São Paulo: Atheneu.

3. Kwak J, Lamprecht C. A Review of the Guidelines for the Evaluation and Treatment of Congenital Syphilis. Pediatrics Ann. [Internet]. 2015; 44(5):108-14. https://doi.org/10.3928/0090448120150512-10

4. World Health Organization. Investment case for eliminating mother-to-child transmission of syphilis: promoting better maternal and child health and stronger health systems [Internet]. Geneva: World Health Organization; 2012. Disponível em: https://portaldeboaspraticas.iff.fiocruz.br/wp-content/ uploads/2020/03/9789241504348_eng.pdf

5. Ministério da Saúde (Brasil). Secretaria de Vigilância em Saúde. Sífilis 2020 [Internet]. Boletim Epidemiológico. 2020;VI(01):1-44. Disponível em: http://www.aids.gov.br/pt-br/pub/2020/boletimsifilis-2020

6. Ministério da Saúde (Brasil). Secretaria de Vigilância em Saúde. Diretrizes para controle da sífilis congênita: manual de bolso [Internet]. 2a. ed. Brasília: Ministério da Saúde; 2006. Disponível em: https://bvsms.saude.gov.br/bvs/publicacoes/manual sifilis bolso.pdf

7. Cohen SE, Klausner JD, Engelman J, Philip S. Syphilis in the modern era: an update for physicians. Infect Dis Clin North Am. 2013;27(4):705-22. https://doi.org/10.1016/j.idc.2013.08.005

8. Andrade BS, Abi Rached CD. Prevenção da transmissão vertical da sífilis congênita: promoção da qualidade da assistência prestada à parturiente. Int. J. Health Manag. Rev [Internet]. 2017;3(2). Disponível em: https://ijhmreview.org/ijhmreview/ article/view/123/60

9. Ministério da Saúde (Brasil). Secretaria de Vigilância em Saúde. Guia de Vigilância em Saúde [Internet]. Brasília (DF): Ministério da Saúde; 2014. Disponível em: https://www.saude.mg.gov.br/ images/documentos/guia_vigilancia_saude_2014.pdf
10. Silva LCVG, Teodoro CJ, Silva JK, Santos DAS, Olinda RA. Perfil dos casos de sífilis congênita em um município do sul de Mato Grosso. J Health NPEPS [Internet]. 2017;2(2):380-390. Disponível em: https://periodicos.unemat.br/index.php/jhnpeps/article/ view/2656/2148

11. Marques JVS, Alves BM, Marques MVS, Arcanjo FPN, Parente CC, Vasconcelos RL. Perfil epidemiológico da sífilis gestacional: clínica e evolução de 2012 a 2017. Sanare. 2018;17(2):13-20. https://doi.org/10.36925/sanare.v17i2.1257

12. Cavalcante PAM, Pereira RBL, Castro JGD. Sífilis gestacional e congênita em Palmas, Tocantins, 2007-2014. Epidemiol. Serv. Saúde. 2017;26(2):255-64. https://doi.org/10.5123/S167949742017000200003

13. Leal MC, Gama SGN, Pereira APE, Pacheco VE, Carmo CN, Santos RV, et al. A cor da dor: iniquidades raciais na atenção pré-natal e ao parto no Brasil. Cad. Saúde Públic. 2017;33(supl 1):e00078816. https://doi.org/10.1590/0102-311X00078816

14. Lima VC, Mororó RM, Martins MA, Ribeiro SM, Linhares MSC. Perfil epidemiológico dos casos de sífilis congênita em um município de médio porte no nordeste brasileiro. J. Health Biol. Sci. 2017;5(1):56-61. http://dx.doi.org/10.12662/2317-3076jhbs. v5i1.1012.p56-61.2017

15. Rodríguez I, Hernández Y. Sexually transmitted diseases during the COVID-19 pandemic: A focus on syphilis and gonorrhoea in Cuba. Public Health Pract. 2021;2:100072. https:// doi.org/10.1016/j.puhip.2020.100072

16. Latini A, Magri F, Donà MG, Giuliani M, Cristaudo A, Zaccarelli M. Is COVID-19 affecting the epidemiology of STIs? The experience of syphilis in Rome. Sex Transm Infect. 2021;97(1):78. http:// dx.doi.org/10.1136/sextrans-2020-054543

17. Di Buduo A, Atzori L, Pilloni L, Perla S, Rongioletti F, Ferreli C. Don't forget about syphilis: sexually transmitted diseases during COVID-19 pandemic. J Public Health Res. 2020;9(4):2040. https:// doi.org/10.4081/jphr.2020.2040

18. Souza GKO, Esquivel VAR, Barros CS, Rabelo MF, Jesus ÉLSM, Paz OS, et al. Perfil epidemiológico dos casos de gestantes com sífilis no estado da Bahia: 2014 a 2019. Rev Eletrônica Acervo Saúde. 2021;13(2):e6254. https://doi.org/10.25248/reas. e6254.2021

19. Oliveira TAK, Morais LE, Leitão JMSR, Verde RMCL, Nascimento $\mathrm{MH}$, Oliveira TR, et al. Caracterização da sífilis em gestantes no município de Codó - Maranhão no período de 2012 a 2017. Rev Eletrônica Acervo Saúde. 2019;(Supl 19):e236. https://doi. org/10.25248/reas.e236.2019

20. Saraceni V, Pereira GFM, Silveira MF, Araujo MAL, Miranda AE. Vigilância epidemiológica da transmissão vertical da sífilis: dados de seis unidades federativas no Brasil. Rev Panam Salud Publica. 2017;41:e44. https://doi.org/10.26633/RPSP.2017.44 
21. Macêdo VC, Romaguera DLM, Ramalho MOA, Vanderlei LCM, Frias PG, Lira PIC. Sífilis na gestação: barreiras na assistência pré-natal para o controle da transmissão vertical. Cad Saúde Coletiva. 2020;28(4):518-28. https://doi.org/10.1590/1414462X202028040395

22. Guimarães TA, Alencar LCR, Fonseca LMB, Goncalves MCC, Silva MA. Sífilis em gestantes e sífilis congênita no Maranhão. Arq. ciênc. saúde. 2018;25(2):24-30. https://doi.org/10.17696/2318$\underline{3691.25 .2 .2018 .1023}$
23. Conceição HN, Câmara JT, Pereira BM. Análise epidemiológica e espacial dos casos de sífilis gestacional e congênita. Saúde Debate. 2019;43(123):1145-58. https://doi.org/10.1590/01031104201912313

24. Figueiredo DCMM, Figueiredo AM, Souza TKB, Tavares G, Vianna RPT. Relação entre oferta de diagnóstico e tratamento da sífilis na atenção básica sobre a incidência de sífilis gestacional e congênita. Cad Saúde Pública. 2020;36(3):e00074519. https://doi. org/10.1590/0102-311X00074519 